\title{
Stock Market Crashes in Australia: A Brief
}

\section{Technical Note}

\section{John Christie ${ }^{1}$}

\begin{abstract}
This paper analyses the three stock market crashes in Australia which have occurred since the All Ordinaries Index was established in 1980. The index behaves in an approximately exponential manner leading up to each market crash and this behaviour can be interpreted as a sign of a looming market crash.
\end{abstract}

JEL classification: G20

Keywords: Stock Market Crashes, Australia

\footnotetext{
${ }^{1}$ University of South Australia, Australia. John.Christie@unisa.edu.au
} 
The All Ordinaries Index was established on January 1, 1980, at the value of 500 points and it is the broadest index on the Australian Securities Exchange (ASX). History shows that when the Australian stock market crashes that the All Ordinaries Index recovers to increase above its previous record high value. We will consider three market crashes in this paper : the crash of 1987, the crash due to the Global Financial Crisis, and the crash due to the coronavirus pandemic.

In the crash of 1987, the All Ordinaries Index had a loss of approximately $49.42 \%$ from its record high of 2312.4 points during trading on September 21, 1987, to reach a low of 1169.6 points during trading on February 10, 1988. Hence, it took approximately 5 months from the high point to the low point, and it then took approximately 72 months for the index to move above 2312.4 points.

In the Global Financial Crisis which began in 2007, the All Ordinaries Index reached a record high of 6873.2 points during trading on November 1, 2007, and had a loss of approximately $55.03 \%$ to reach a low of 3090.8 points during trading on March 10, 2009. Hence, it took approximately 16 months from the high point to the low point, and then it took approximately 124 months for the index to reach the next record high.

In the current coronavirus pandemic, the All Ordinaries Index reached a record new high of 7289.7 points during trading on February 20, 2020, and then had its quickest ever crash to reach a low point of 4429.1 points during trading on March 23, 2020, a loss of approximately $39.24 \%$. History shows that the stock market will eventually recover lost ground but how long will this take? It would be forecast to take at least a year, as vaccines have been found which will be widely available in Australia starting from late February, 2021.

Are there any warning signs before a stock market crash? Before the three crashes, the All Ordinaries Index grew approximately exponentially, as can be seen in Figure 1 below.

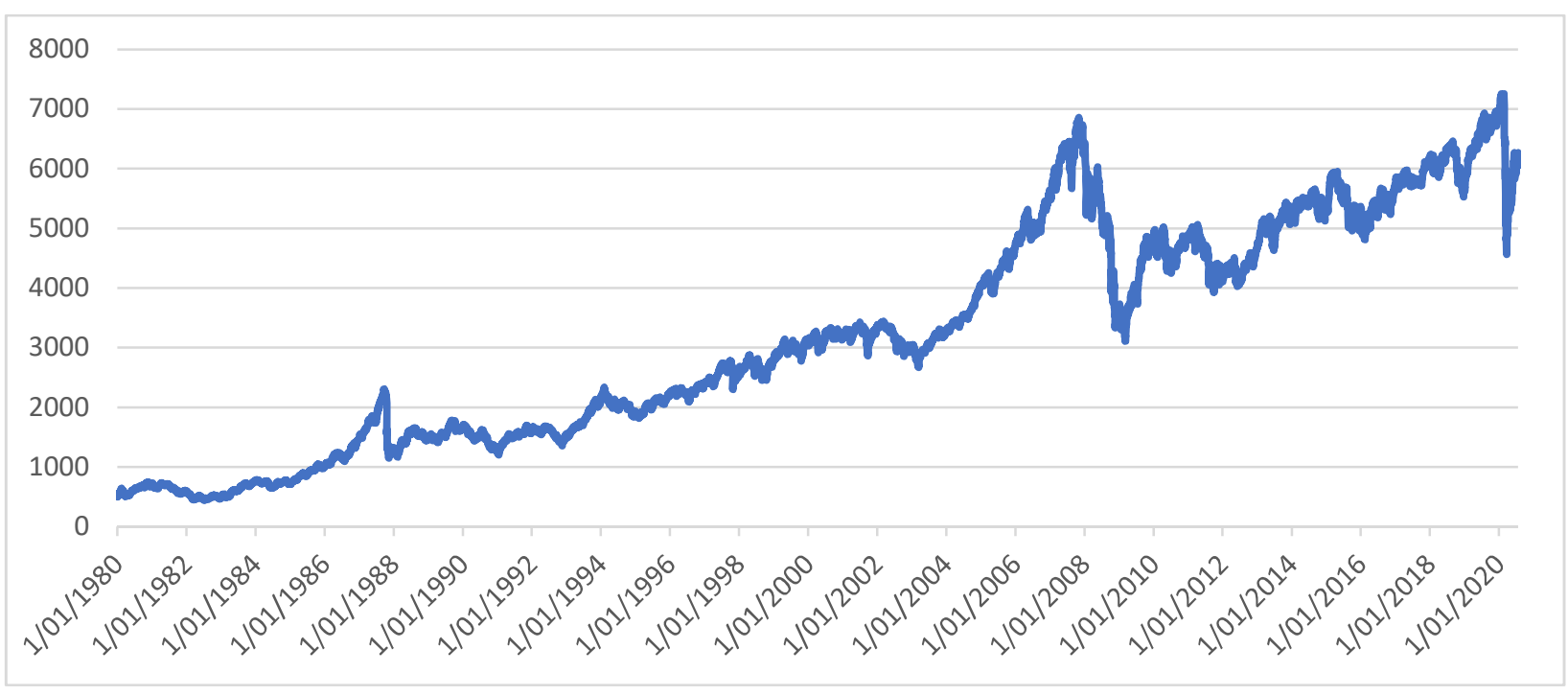

Figure 1. Plot of the All Ordinaries Index from January 1, 1980, to July 22, 2020.

This can be seen more conclusively when the logarithm of the All Ordinaries Index is plotted as shown in Figures 2-4 below. 


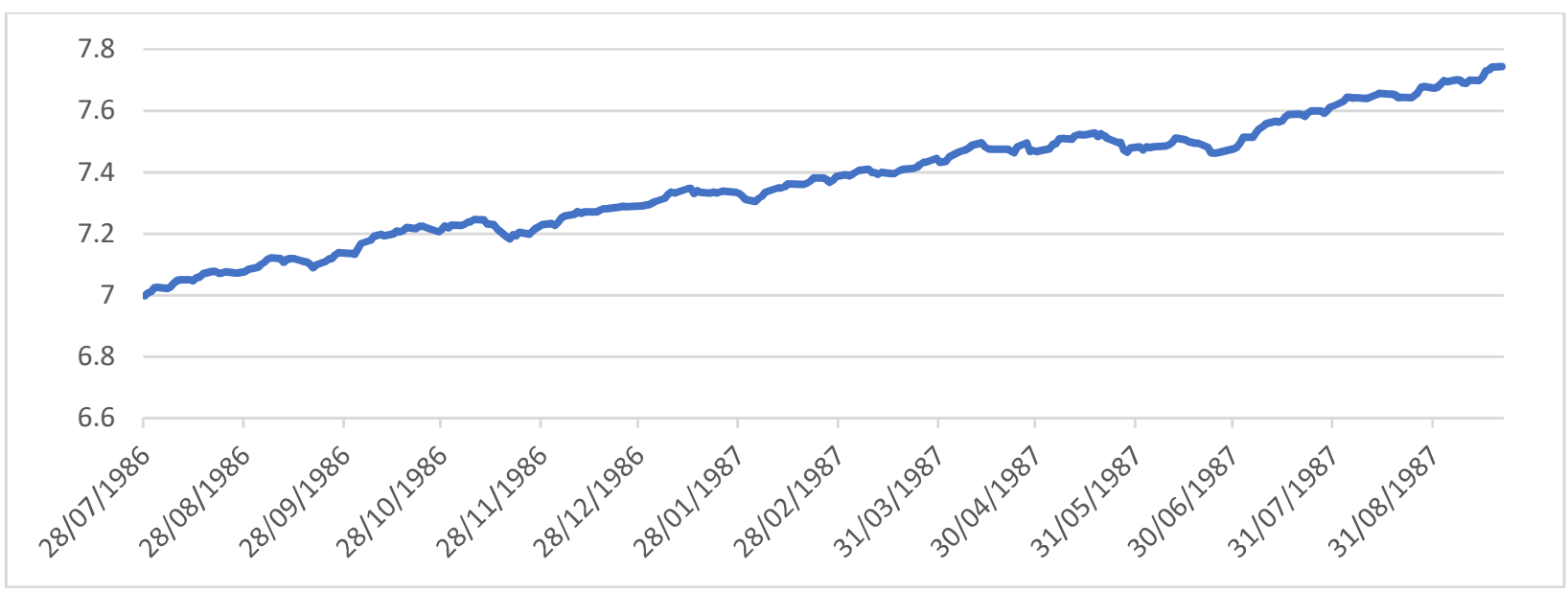

Figure 2. Plot of the logarithm of the All Ordinaries Index from July 28, 1986, to September 21, 1987.

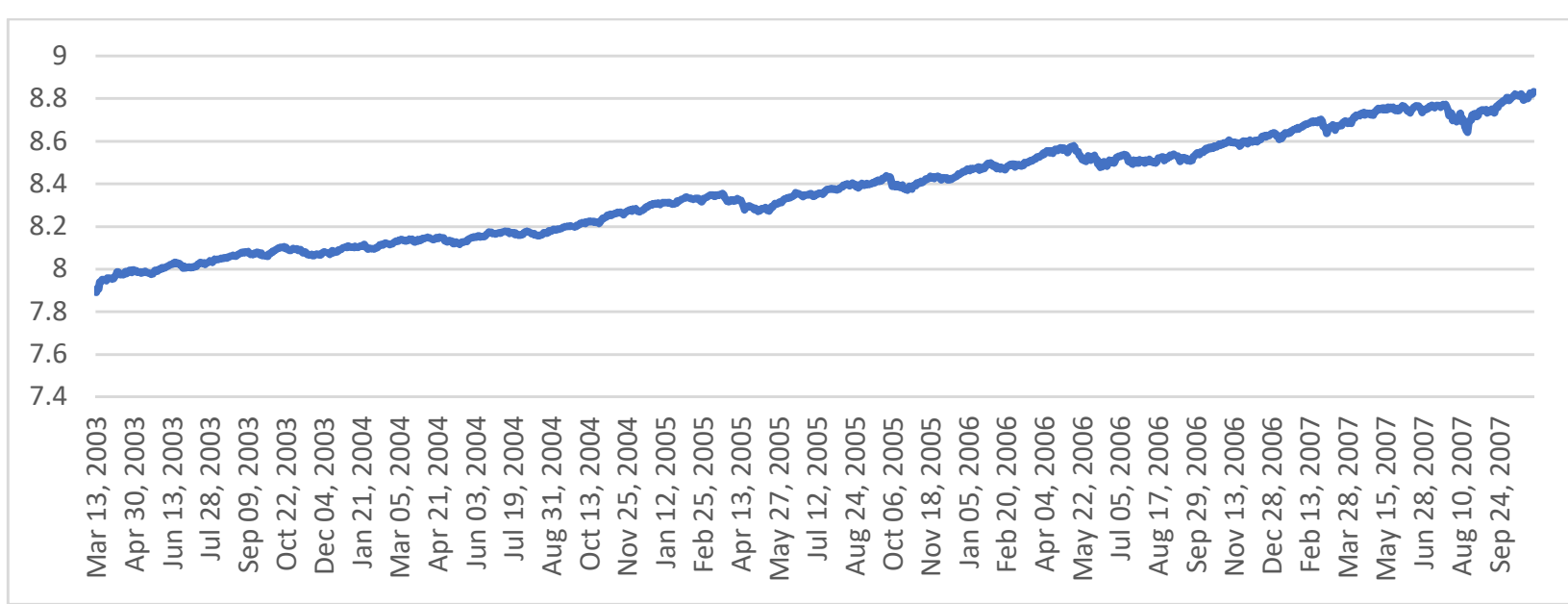

Figure 3. Plot of the logarithm of the All Ordinaries Index from March 13, 2003, to November 1, 2007.

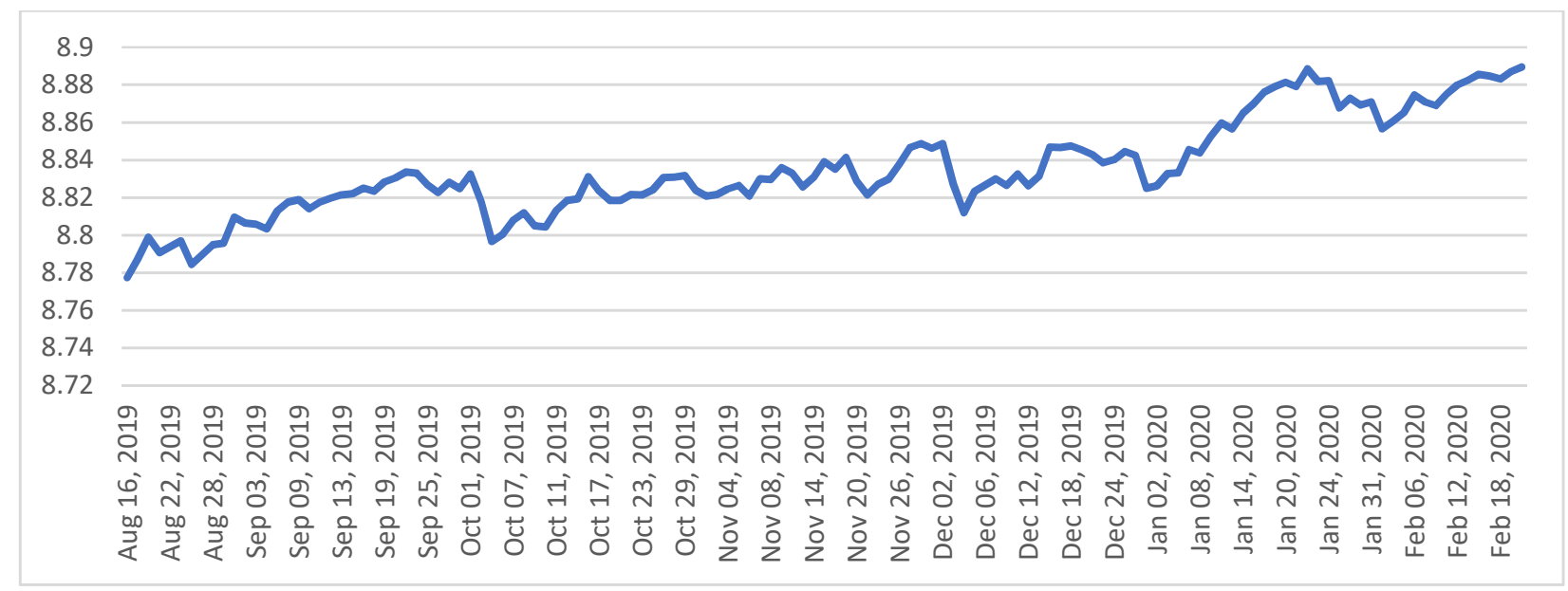

Figure 4. Plot of the logarithm of the All Ordinaries Index from August 16, 2019, to February $20,2020$. 
Each of the three graphs is approximately a straight line which implies approximate exponential growth for the All Ordinaries Index, before a stock market crash. It can be concluded that when the index grows exponentially for an extended period of time, then the stock market will eventually crash, but it is not possible to predict the length of this period of time. This exponential growth for the All Ordinaries Index can be interpreted as a warning sign of a future market crash. 